\title{
Research on Digital Library Based on Cloud Computing
}

\author{
Xueyan Wang ${ }^{1, a}$, Hongmin Wang ${ }^{2, b}$ \\ ${ }^{1}$ Liaoning Institute of Science and Engineering, Jinzhou, P.R. China \\ ${ }^{2}$ College of Information Science and Technology, Bohai University, Jinzhou, P.R. China \\ a29853638@qq.com, b'whm8665@yeah.net
}

Keywords: cloud computing; digital library; virtualization; service layer; technology

\begin{abstract}
With the rapid development of the Internet and cloud computing applications, the construction of a digital library in the cloud computing environment has been put on the agenda. Construction and management of digital libraries using cloud computing technology, can effectively utilize resources, reduce costs, achieve better information sharing, and improve the service function of the library. In this paper, studies digital library based on cloud computing. Firstly, introduces the characteristics and establish principles of digital library based on cloud computing; secondly, elaborated the concept and work principle of cloud computing; then, describes the key technology of cloud computing, and a detailed introduction of virtual storage technology in digital library; and finally introduced cloud computing digital library service model, including cloud software as a service layer (SaaS), cloud platform as a service layer (PaaS), cloud infrastructure as a service layer (IaaS).
\end{abstract}

\section{Introduction}

The adoption of cloud computing technology for library, is described as: "no need for each library is equipped with a large room facilities, librarians can use the same computer with the readers, as long as connect to the Internet at the library, each machine can be carried out transaction processing of library business" [1]. Cataloguing data already in the "cloud", by OCLC or other united catalogue center according to the publisher's cataloging data service to convert, publishers and several large catalog center directly on the "data cloud" to operate, library log in to the system of resource issuers, After ordering success, the corresponding data into library collection system, if it is a digital resource, license management also simultaneously; the reader can use any authentication information to register or log in system, and obtain lending, consulting and other services; digital library resources are distributed on the "cloud", various cloud service providers offer different storage services; libraries can take advantage of cloud computing platform for the integration of digital resources, organization, association, navigation or even visual service. Behind each library are interconnection network as a support, to achieve interlibrary loan and resource sharing through a network protocol.

Book has a large amount of information, the need for a large storage space, and information resource duplication between the library and library, resulting in a lot of information, so that the information garbage excessive growth, at the present stage. If these databases with cloud computing thinking thought it unified placement, unified management, information resources that the library will be able to realize that one hundred percent of the share [2]. Establish the principle of digital library: to construct a cloud service platform of digital library based on user as the center as a fundamental principle, comprehensive considering a variety of factors, efficiency, economy, practicality and so on, through the application of cloud computing technology, fully integrated digital information resources of library, and ultimately realized digital library under cloud platforms and cloud alliance [3]. In the cloud computing environment, digital library management and service, to a large extent, available to users through the Internet, the library does not need to invest a lot of money and then purchase a digital library infrastructure and resources, but does not require specialized technical personnel to manage and maintain the library system, the use of digital libraries terminal equipment connected to the Internet, to achieve the retrieval of collections of 
resources, cataloging, lending and other management efforts, and provide the necessary library services to users via the Internet.

\section{Cloud Computing Concept and Principle}

Cloud computing concept was proposed by Google, refers to the open standards and service as the foundation, it takes the Internet as the center, to provide a safe, fast, convenient data storage and network computing service. This service is the product of these traditional computer technology and network technology integration developments. Traditional computer technology have grid computing, distributed computing, parallel computing, utility computing, network storage, virtualization, load balancing. It aims to integrate multiple networks relatively low-cost computing entities into a perfect system with powerful computing capabilities, and with SaaS, PaaS, IaaS, MSP and other advanced business model to powerful computing power distributed to terminal user [4]. The core concept of cloud computing is through the continuous improvement of the "cloud" processing power, thereby reducing the processing burden on the user terminal, user terminal eventually reduced to a simple input and output devices, and can enjoy the "cloud," a powerful computing capacity according the required, constitute a pool of computing resources on demand service to users.

The working principle of cloud computing, according to different manufacturers and engineers to define, cloud computing model can be divided into software as a service, utility computing, network services of cloud computing, managed service providers, service business platforms and network integration model [5]. Working principle is by distributed on the computer of the network to simulate large-scale supercomputer to calculate. The running of library management data centers is similar with network running, so that the library service resources can be can be switched to the desired application, depending on different demand access to different computers and storage systems, cloud computing working principle is shown in Fig. 1.

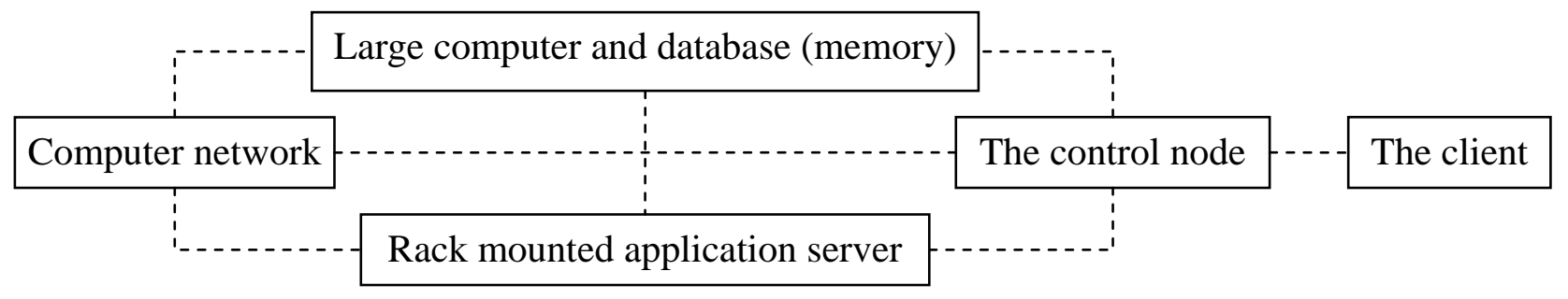

Fig. 1. The working principle of cloud computing

\section{The Key Technology of Cloud Computing}

Cloud computing technology epitomizes the characteristic features of information resources services in network era, the key support technology mainly has the following several kinds: virtualization technology. Cloud computing model to build inseparable virtualization support technology, the advantages of virtualization is simple in its main deployment, effective management of servers and storage, clients computer will be available to users; automation technology. Automation technology is the foundation of cloud computing infrastructure, can achieve real time and on demand to carry on effective management to the backstage resources; the cloud database, cloud computing era, cloud database to replace the relational database, enabling it to run in a distributed environment with multiple servers in different locations; cloud operating system, which uses cloud computing and cloud storage operating system; cloud security. Cloud computing technology can achieve that is not exposed to the user original data, at the same time, according to user needs to deal with the corresponding data [6].

Virtual storage technology in digital library, in the cloud storage environment, physical distribution various types of storage systems and devices can be into a single virtual storage system that provides a unified, transparent, secure access and management mechanisms. So that the physical distribution storage server can independently access to the cloud computing, to form a 
unified access interface through storage virtualization technology of intermediate layer [7]. The virtual storage system in the original system increases the virtualization layer, a plurality of storage units is abstracted into a virtual storage pool, storage users through the interface provided by the virtualization layer proposed virtual $\mathrm{I} / 0$ requests to virtual storage pools, the virtualization layer processing the request, the corresponding request mappings to specific storage unit, which can reduce administrative overhead of storage system, will unify multiple independent storage units into a single logical storage systems, effectively implement multiple storage system data sharing. Digital library using NAS/SAN storage mode can only achieve the separation of physical level between storage resources and computing resources, and cannot realize the separation of logic level.

\section{Service Model of Digital Library Based on Cloud Computing}

Digital library application model based on cloud computing is mainly composed of infrastructure services layer, platform services layer, application service layer and cloud client components. Infrastructure services layer which is cloud computing digital libraries provide users with cloud services, is the bottom layer on the whole cloud computing digital library model architecture application system, the main function of this layer is some function modules that are processed through the virtualization technology, and in the architecture hardware resources for effective integration, the layer is mainly composed of two parts of service function[8]: the first part is through distributed clustering technology, abstract technology and virtualization technology on the Internet network, host hardware equipment and storage devices for processing, after processing, hardware and function module is formed a whole through the cluster, so formed the infrastructure service layer of the entire cloud computing digital library architecture, users need only to simply issue a corresponding instruction information, can obtain the "cloud" resource information they need, users do not need to know specific information from which device; the second part is established on the basis of the first part, its main function is to provide users with flexible computer management, load management, data resource backup and storage management, these service interface function, which makes the digital library based on cloud computing infrastructure layer service more flexible and more convenient. Generally speaking, cloud computing provided services architecture by virtualization, shown in Fig. 2.

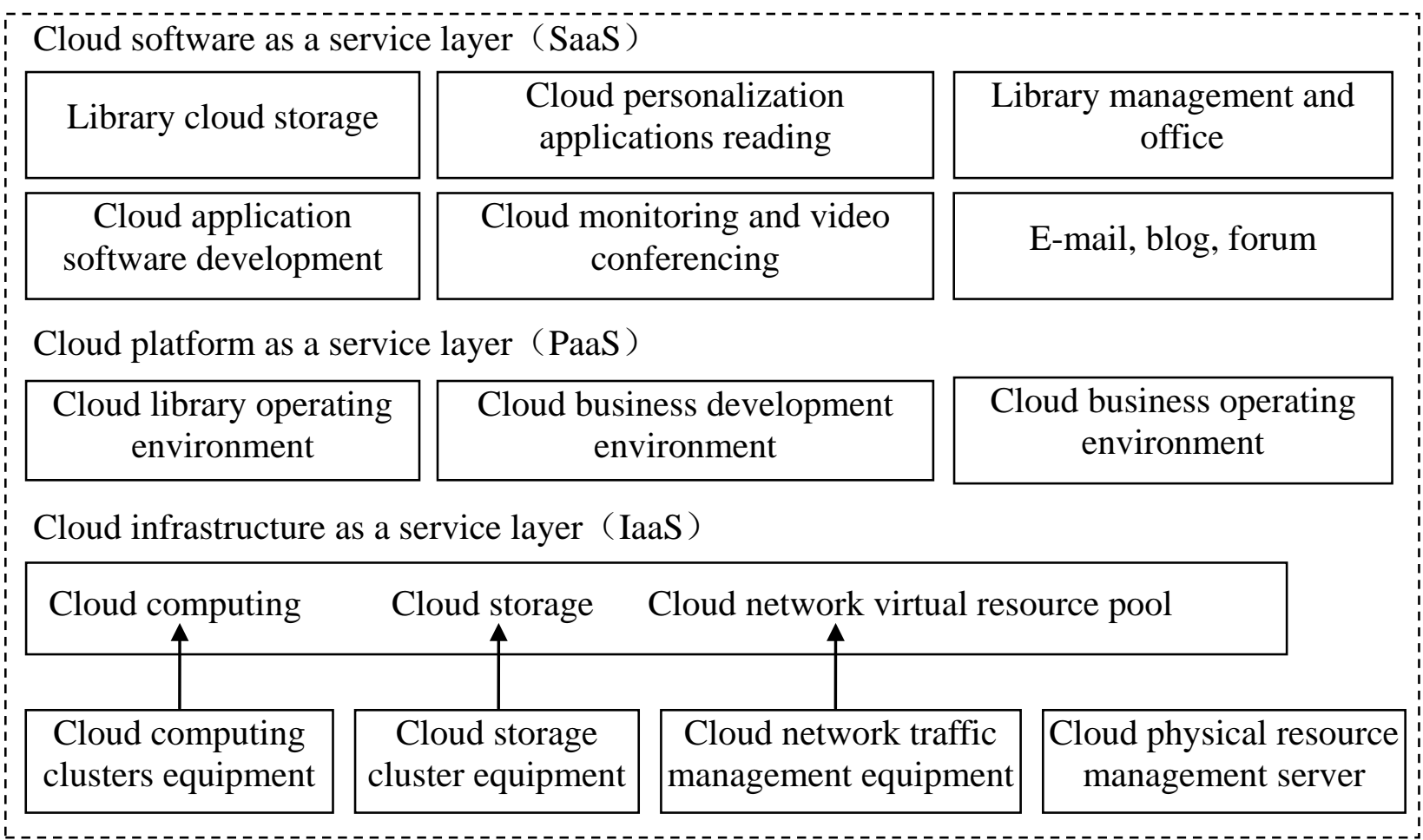

Fig. 2. Digital library service model under cloud computing environments 
As can be seen from Fig. 2, each layer of cloud service platform for digital library can take advantage of corresponding cloud services to build, and can achieve the appropriate service offered by digital libraries [9]. Such as the use of cloud computing technology software as a service (SaaS) to build cloud library automation integrated management system, a unified retrieval platform, and other application software; the use of platform as a service (PaaS) to build a library database services and application development environment services platform; use infrastructure as a service (IaaS) to build a library storage and data center applications environments.

\section{Conclusion}

Powerful computing and storage functions of cloud computing platform, provides a good platform for the integration of resources, integration, sharing of digital library. The ultimate aim of digital library is for readers to service. Through cloud computing model, between the relevant library can work together to build information sharing space of library, so many libraries can share the infrastructure by a large number of systems connected together to form, without having to update the associated hardware, library operating costs at the same time greatly reduced, at the same time efficiency is greatly improved. In addition, under cloud computing environments each of library information resources clients is not required their own to configure resources, but the common use of cloud services platform, to achieve the maximum of resources utilization. In the cloud computing environment, the cloud service providers to provide daily maintenance of server, library information resources management departments do not have to pay an additional fee. For the library, the cloud computing system capabilities and features exactly what they need.

\section{Acknowledgement}

This work is supported by social science fund project of Liaoning province (L13DTQ01).

\section{References}

[1] Y. Luo, "On the Application of Cloud Computing to the Digital Library," Journal of Chongqing Three Gorges University, vol. 27, no.130, pp.147-149, 2011.

[2] X. J. Lu, "Cloud Computing and the Building of Digital Information Resources in the Future Library," Journal of the Library Science Society of Sichuan, vol. 31, no. 2, pp.23-24, 2009.

[3] J. Y. Wang, "Cloud Computing Technology based on Digital Library of Cloud Services Platform Architecture," Jilin University, 2011.

[4] H. M. Jin, "Application of Cloud Computing in the Construction of Digital Resources in Library," Gansu Science and Technology, vol. 27, no. 7, pp. 84-86, 2011.

[5] H. J. Liu, "Cloud Computing Applications in Digital Library Construction and Service," Sci-Tech Information Development and Economy, vol. 20, no. 17, pp. 21-23, 2010.

[6] T. T. Yang, "Exploration of the Digital Library Construction under Cloud Computing Technology," Journal of Xinjiang Normal University (Natural Sciences Edition), vol. 32, no. 2, pp.54-57, 2013.

[7] X. Q. He, "Research on Libraries digital resources Storage based on Cloud Computing," Information Research, vol. 24, no. 12, pp.92-94, 2010.

[8] Y. Zhang, "The Application Model Research of Digital Library Based on Cloud Computing," Computer Programming Skills and Maintenance, vol. 20, no. 10, pp.47-48, 2013.

[9] X. Yu, "Research on the Cloud Services Platform Architecture of Digital Library Based on Cloud Computing Technology," Information Science, vol. 29, no. 7, pp. 1049-1053, 2011. 